\title{
THE EVALUATION CRITERION FOR COLOR IMAGE SEGMENTATION ALGORITHMS
}

\author{
Peter Lukáč — Róbert Hudec - Miroslav Benčo \\ Zuzana Dubcová - Martina Zachariášová — Patrik Kamencay *
}

\begin{abstract}
Image segmentation is first and very important step in image analysis. The main idea of image segmentation is to simplify and change image into easier and meaningful form to analyze. Image segmentation is process, which locate objects in image. Many segmentation algorithms have been created for different applications. The algorithms are used in traffic applications, army applications, web applications, medical applications, studying and many others. In present time, do not exist restful objective methods to evaluate segmentation algorithms. This paper presents evaluation criterion based on measurement of precision of boundary segmentation. Moreover, the automatic segmentation algorithms in comparison with human segmentation results were tested. Four most used image segmentation algorithms, namely, Efficient graph based, K-means, Mean shift and Belief propagation are compared by designed criterion. The criterion computes three evaluation parameters like precision, recall and $F_{1}$ and the results are presented in the tables and graphs at the end of the paper.
\end{abstract}

K e y w or d s: image, segmentation, algorithm, evaluation criterion

\section{INTRODUCTION}

In computer vision, the term image segmentation presents process of partitioning an image into regions (segments) that cover it. The goal for the regions is to represent meaningful areas of the image [1].

The result of image segmentation is a set of regions that collectively cover the entire image, or a set of contours extracted from the image. Pixels in the regions are similar or different with respect to some characteristic or features, such a color, intensity, or texture. Pixels in similar regions have resembling attributes and pixels in adjacent regions have different attributes [3].

Image segmentation is often used as a basic step in many techniques associated with the analysis of image contents (Fig. 1). The efficiency of segmentation methods is in the content based image retrieval system (applications like digital image databases or multimedia digital libraries), in medical imaging, video coding, industrial animation and many others $[4,5]$.

\section{IMAGE SEGMENTATION ALGORITHMS}

The image segmentation algorithms can be grouped into three main categories $[2,3]$ :

- clustering based methods,

- region growing methods,

- edge detection methods.

In clustering based methods, pixels of the image are grouped together by a divisive or cumulative criterion involving only their values in the color space. In region growing methods, pixels are progressively connected in a region and they have the same mean and variance values. In edge detection methods, regions are identified starting from their contours by identifying points, where they have different properties.

In this paper, efficient graph based, k-means, mean shift and belief propagation algorithms are presented.

\subsection{Efficient graph based algorithm}

The EGA (Efficient Graph based Algorithm) presented in [6] deals about problem in terms of a graph $G=(V, E)$ where nodes $v_{i} \in V$ represents pixels in the image, and the edges $\left(v_{i}, v_{j}\right) \in E$ connect certain pairs of neighboring nodes. Each edge $\left(v_{i}, v_{j}\right)$ has responding weight $w\left(v_{i}, v_{j}\right)$ that is nonnegative dissimilarity measure between connected nodes by the edge ( $e g$ the difference in color, location, intensity, motion etc). There are several techniques to correct merge two pixels. One of most popular is merging pixels via its similarity or dissimilarity. Thus, edges between two vertices in the same segment should have low weights and high weights for edges between two vertices in different segments [6].

EGA has two important tasks, namely, definition of difference between two components or segments and the definition of threshold function. The algorithm starts with the step, where each segment contains one pixel only. In the next step, segments are iterative merged by the following condition

$$
\begin{aligned}
& \operatorname{Diff}\left(C_{1}, C_{2}\right) \leq \operatorname{Int}\left(C_{1}\right)+T\left(C_{1}\right), \\
& \operatorname{Diff}\left(C_{1}, C_{2}\right) \leq \operatorname{Int}\left(C_{2}\right)+T\left(C_{2}\right),
\end{aligned}
$$

\footnotetext{
* Department of Telecommunications and Multimedia, University of Žilina, Univerzitná, 01026 Žilina, Slovakia, \{peter.lukac, robert.hudec, miroslav.benco, zuzana.dubcova, martina.zachariasova, patrik.kamencay\}@fel.uniza.sk
} 


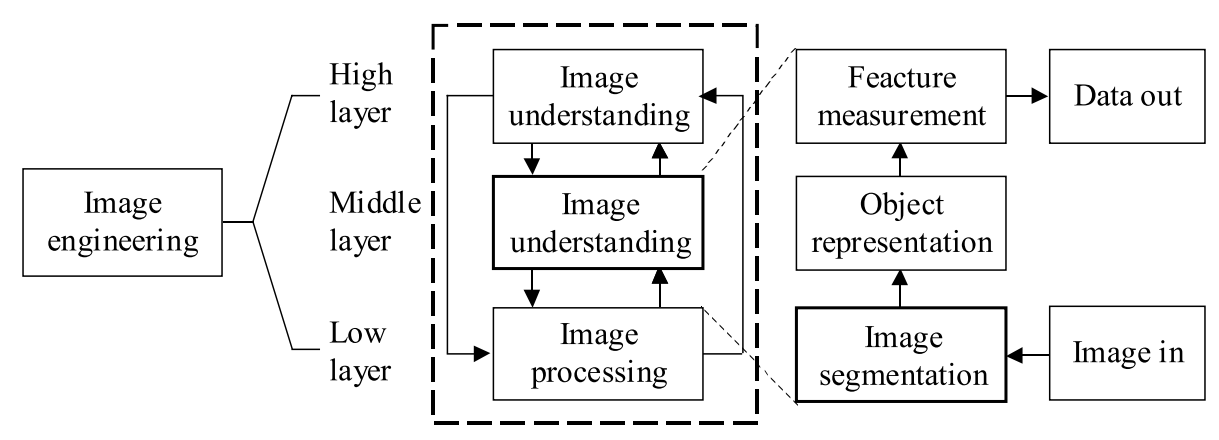

Fig. 1. Image engineering and image segmentation

where $\operatorname{Diff}\left(C_{1}, C_{2}\right)$ is the difference between $C_{1}$ and $C_{2}$ components, $\operatorname{Int}\left(C_{1}\right)$ and $\operatorname{Int}\left(C_{2}\right)$ are internal differences of $C_{1}$ and $C_{2}$ components, $T\left(C_{1}\right)$ and $T\left(C_{2}\right)$ are threshold functions of $C_{1}$ and $C_{2}$ components [6].

The threshold function controls the level of merging two segments, where in order to boundary detection the difference between two segments must be bigger than their internal difference. Threshold function is defined as follows

$$
T(C)=\frac{k}{|C|},
$$

where $|C|$ presents the size of component $C, k$ parameter is constant, which manages size of the components. For small segments is required stronger evidence of a boundary. Larger $k$ causes a preference for larger segments, smaller segments are allowed when there is a sufficiently large difference between them [6].

\subsection{K-means algorithm}

KA (K-means Algorithm) is statistical clustering algorithm. Data clustering is method which creates groups of objects (clusters). KA like other clustering algorithms is based upon the index of similarity or dissimilarity between pairs of data components. KA is iterative, numerical, non- deterministic and unsupervised method. This type of algorithm is popular for simplicity for implementation and it is commonly used for grouping pixels in images $[7,8]$.

$$
\begin{gathered}
M_{k}=\left\{U \in V_{K N} \mid u_{i k} \in\{0,1\} \forall i, k ;\right. \\
\left.\sum_{i=1}^{K} u_{i k}=1 \forall k ; 0<\sum_{k=1}^{K} u_{i k}<N \forall i\right\},
\end{gathered}
$$

Let $X=\left\{x_{1}, x_{2}, \ldots, x_{n}\right\} \subset R^{p}$ be a finite set of data where $n$ is the number of data items and $R^{p}$ is $p$-dimensional Euclidean space. Let $V_{K N}$ be the set of matrices $(K \times N, 2 \leq K<N)$, where $K$ is the number of clusters. $K$-th partition of $\mathrm{X}$ is defined in equation (3), where $u_{i k}=1$ denotes that component $x_{k}$ belongs to the cluster $i, u_{j k}=0$ denotes that component $x_{k}$ is out of the cluster $j$. The objective function $J_{K}$ is

$$
J_{K}(U, V)=\sum_{i=1}^{K} \sum_{k=1}^{N} u_{i k} d_{i k}^{2},
$$

where $V=\left\{v_{1}, v_{2}, \ldots, v_{K}\right\}, v_{i} \in R^{p}, 1 \leq i \leq K$, denotes set of $K$ clusters and $d_{i k}=\left\|x_{k} v_{i}\right\|$ denotes Euclidean distance between component $x_{k}$ and cluster $v_{i}$. Optimal $K$ clusters of $X$ is produced by minimization of objective function $J_{K}(U, V)$ [9].

\subsection{Mean shift algorithm}

The MSA (Mean Shift Algorithm) is non-parameter iterative algorithm. Let $x_{i}, i=1,2, \ldots, n$ be a set of points in $d$-dimensional space $R^{d}$. The number of points $x_{i}$ belonging to $d$-dimensional area near to $x$ with edge length $h$ is given by

$$
\sum_{i=1}^{n} K\left(\frac{x-x_{i}}{h}\right)
$$

where $K(x)$ is a kernel or window function. Thus, the kernel density estimation has the following form

$$
\hat{f}_{h, K}=\frac{1}{n h^{d}} \sum_{i=1}^{n} K\left(\frac{x-x_{i}}{h}\right) .
$$

Assuming a radial symmetric kernel, where $K(x)=$ $c_{k, d} k\left(\|x\|^{2}\right), k(x)$ is kernel profile and $c_{k, d}$ is normalized constant, the formula (6) can be rewritten to

$$
\hat{f}_{h, k}(x)=\frac{c_{k, d}}{n h^{2}} \sum_{i=1}^{n} k\left(\left\|\frac{x-x_{i}}{h}\right\|^{2}\right) .
$$

Using the estimate as the gradient of the density estimation

$$
\begin{aligned}
\nabla \hat{f}_{h, k}(x)=\frac{c_{k}}{n h^{d}} \sum_{i=1}^{n} \nabla k\left(\left\|\frac{x-x_{i}}{h}\right\|^{2}\right) \\
\quad=\frac{2 c_{k}}{n h^{d}} h^{-2} \sum_{i=1}^{n}\left(x-x_{i}\right) k^{\prime}\left(\left\|\frac{x-x_{i}}{h}\right\|^{2}\right)
\end{aligned}
$$




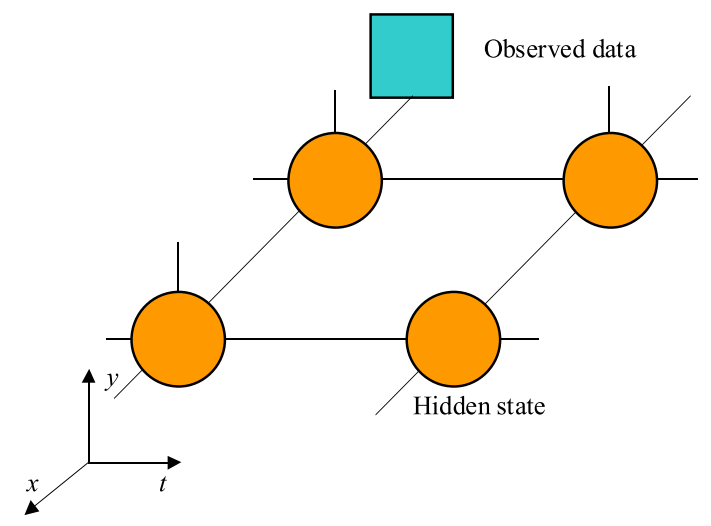

Fig. 2. MRF model

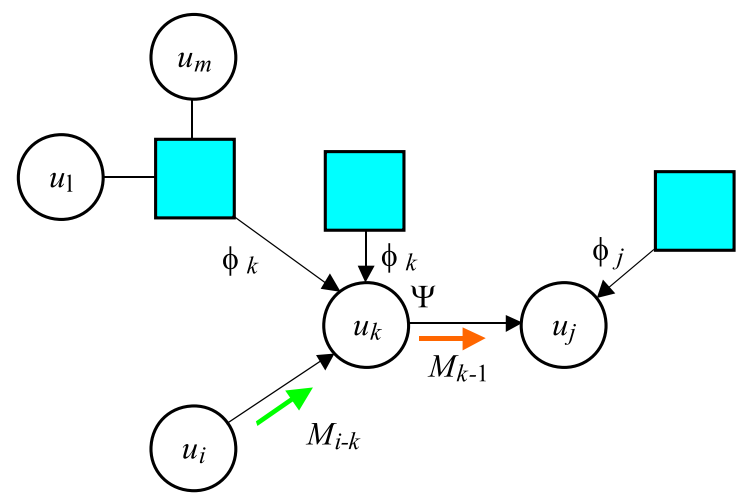

Fig. 3. Belief propagation structure of sending messages

and

$$
g(x)=k^{\prime}(x),
$$

where $g(x)$ is a new kernel function, which is defined as derivate of $k(x)$, becomes

$$
\nabla \hat{f}_{h, k}(x)=\frac{2 c_{k}}{n h^{d}} h^{-2} \sum_{i=1}^{n}\left(x-x_{i}\right) g\left(\left\|\frac{x-x_{i}}{h}\right\|^{2}\right) .
$$

Then

$$
\begin{aligned}
\nabla \hat{f}_{h, k}(x)=\frac{2 c_{k, d}}{n h^{d+2}}[ & \left.\sum_{i=1}^{n} g\left(\left\|\frac{x-x_{i}}{h}\right\|^{2}\right)\right] \\
& \times\left[\frac{\sum_{i=1}^{n} x_{i} g\left(\left\|\frac{x-x_{i}}{h}\right\|^{2}\right)}{\sum_{i=1}^{n} g\left(\left\|\frac{x-x_{i}}{h}\right\|^{2}\right)}-x\right] .
\end{aligned}
$$

From this equation, the mean shift vector can be defined

$$
m_{h, g}(x)=\frac{\sum_{i=1}^{n} x_{i} g\left(\left\|\frac{x-x_{i}}{h}\right\|^{2}\right)}{\sum_{i=1}^{n} g\left(\left\|\frac{x-x_{i}}{h}\right\|^{2}\right)}-x .
$$

The mean shift algorithm is based on iterative computing of mean shift vector and consistent actualizing of kernel position by $[10,11]$

$$
x^{k+1}=x^{k}+m\left(x^{k}\right) .
$$

\subsection{Belief propagation algorithm}

BPA (Belief Propagation Algorithm) is an iterative inference algorithm for graphical models such as MRF (Markov Random Field), which is based on a message passing principle that propagates messages in the network. MRF models are often used for image segmentation, because of ability to capture the context of an image (ie, dependencies among neighboring image pixels) and deal with the noise. A typical MRF model for image segmentation is a graph with two kinds of nodes: hidden nodes (circles in Fig. 2) representing region labels and observable nodes (squares in Fig. 2) representing pixels in image. Edges in the graph depict relationships among the nodes $[12,13]$.

The above model contains only pairwise cliques, then the joint probability is

$$
P\left(u_{1}, \ldots, u_{n}, v_{1}, \ldots, v_{n}\right)=\prod_{i \neq j} \psi_{i, j}\left(u_{i}, u_{j}\right) \prod_{k} \phi_{k}\left(v_{k}, v_{k}\right),
$$

where $u$ and $v$ represent the state node and the data node separately, $\psi$ is the state transition function between a pair of different hidden state nodes and $\phi$ is the measurement function between the hidden state node and observed data node. Number $N$ represents the total number of state or data nodes. Under the squared loss function, the best estimation for node $u_{j}$ is the mean of the posterior marginal probability (minimum mean squared error estimation (MMSE estimation)

$$
u_{j M M S E}=\sum_{j} u_{j} \sum_{k \neq j} P\left(u_{1}, \ldots, u_{N}, v_{1}, \ldots, v_{N}\right) \text {. }
$$

where the inner sum gives the marginal distribution of $u_{j}[13]$.

From time the joint probability involves all the hidden state nodes and data nodes, it is hard to compute the MMSE estimation based on the multivariable probability distribution. However, belief propagation messages are to effective computing the MMSE estimate recursively. Each hidden state node has a belief, which is a probability distribution defining the nodes motion likelihood. Thus the MMSE estimation of one node is computed as follows

$$
u_{j M M S E}=\sum_{j} u_{j} b\left(u_{j}\right)
$$

where

$$
b\left(u_{j}\right)=\phi\left(u_{j}, v_{j}\right) \prod_{k \in \operatorname{Nejghbor}(j)} M_{j}^{k},
$$

is the belief at node $u_{j}$ and $k$ runs over all neighboring hidden state nodes of node $u_{j}$. The belief at node $u_{j}$ is the product of all incoming messages $M$ and the local observed data message $\left(\phi_{j}\left(u_{j}, v_{j}\right)\right)$. The structure of sending messages is shown in Fig. 3. The passed messages specify the distribution of each node neighbors. Equation

$$
M_{j}^{k}=\sum_{k} \psi_{j k}\left(u_{j}, u_{k}\right) b\left(u_{k}\right)
$$

shows how to compute the message from node $u_{k}$ to $u_{j}$. 


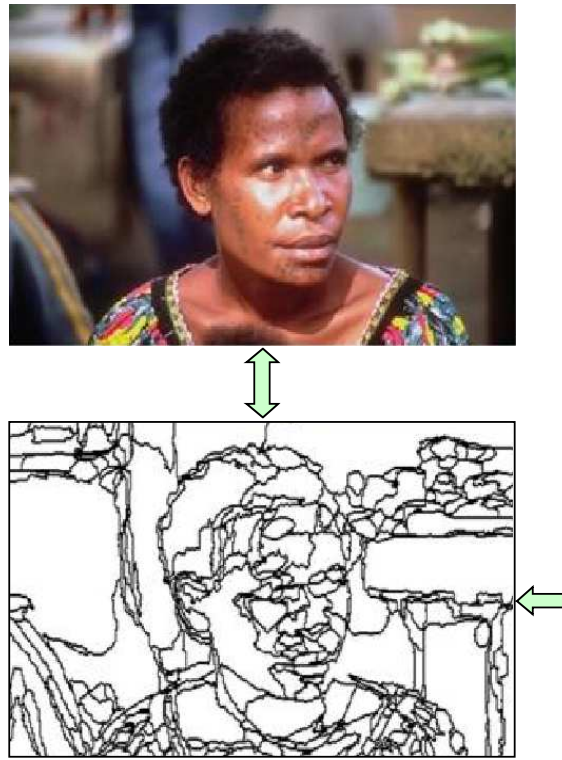

(a)

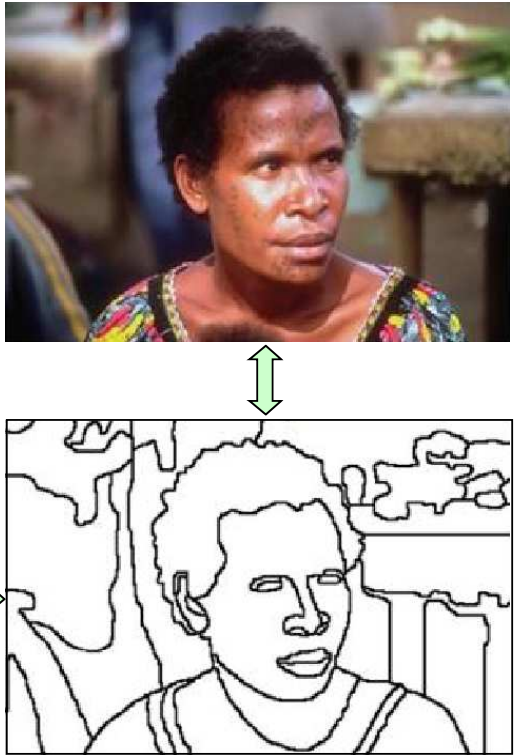

(b)

Fig. 4. Example of segmentations comparison (a) segmentation by algorithm, (b) segmentation by human

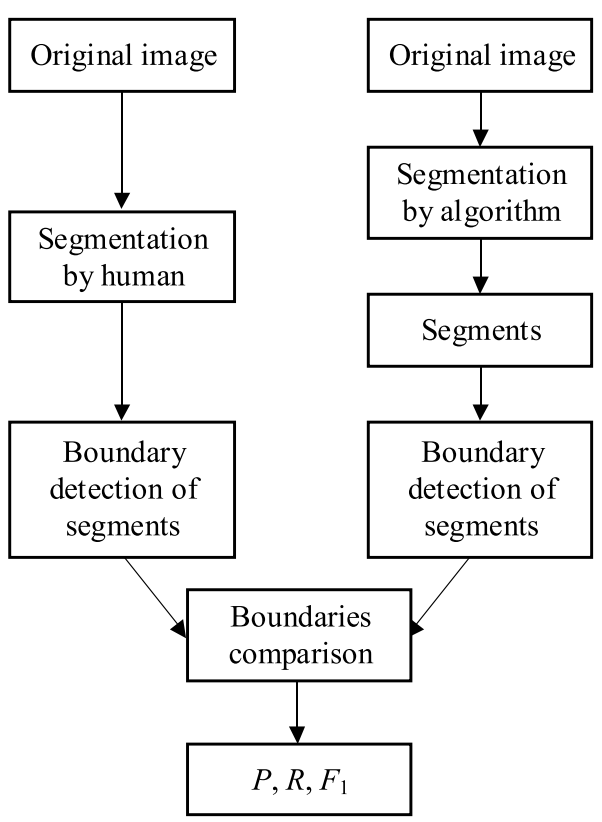

Fig. 5. Principal block diagram of evaluation criterion

Table 1. Averaged results achieved for real images

\begin{tabular}{cccccc}
\hline $\begin{array}{c}\text { Type of } \\
\text { algorithm }\end{array}$ & $\begin{array}{c}\text { Number } \\
\text { of images }\end{array}$ & $\begin{array}{c}P \\
(\%)\end{array}$ & $\begin{array}{c}R \\
(\%)\end{array}$ & $\begin{array}{c}F_{1} \\
(\%)\end{array}$ & Score \\
\hline EGA & & 49.05 & 35.25 & 40.68 & 1 \\
KA & \multirow{2}{*}{100} & 54.08 & 22.27 & 30.81 & 4 \\
MSA & & 58.77 & 29.65 & 38.58 & 2 \\
BPA & & 61.41 & 25.30 & 35.23 & 3 \\
\hline
\end{tabular}

After substituting $b\left(u_{k}\right)$ by equation (17)

$$
M_{j}^{k}=\sum_{k} \psi_{j k}\left(u_{k}, u_{+} j\right) \phi\left(u_{k}, v_{k}\right) \prod_{j \in \operatorname{Nejghbor}(k) / j} M_{k}^{i}
$$

where $i \in N$ Nighbor $(k) / j$ denotes all neighboring nodes of $k$ different from $j$. After multiplying all incoming messages $M$ from neighboring nodes (except from the node $\left.u_{j}\right)$ and the observed data message $\left(\phi_{k}\left(u_{k}, v_{k}\right)\right)$, the product is evolved from the message-sender to the message-receiver by transition function $\psi_{k j}\left(u_{k}, u_{j}\right)$ [13].

\section{SEGMENTATION EVALUATION CRITERION}

Increasing of the developed algorithm brought the task about evaluation criterions. Generally, basic techniques exist for objective evaluation the quality of color image segmentation, analytical and experimental techniques. The analytical technique is based on analysis of algorithms principle, complexity, robustness the algorithms. The experimental technique is used for evaluation, interpretation and results comparison of color image segmentation algorithms. Our evaluation technique is based on the visualized comparison of the result of segmentation algorithm and result by human segmentation.

The evaluation criterion used for experiments and comparison the algorithms, is based on the computing precision $(P)$, recall $(R)$ and $F_{1}[14]$. The parameters $P$, $R$ and $F_{1}$ characterize efficiency of the algorithms. Their computing is based on the results comparison made by algorithm and predicted by human. Example of the comparison is shown in Fig. 4.

The evaluation criterion algorithm, introduced in this article, consists of few basic steps. Segmented images are used as input data. One type of data is achieved by human, the second input data is segmented image by algorithm. The segmentation by human has boundaries of segments as the result of segmentation. Thus in the first step, algorithm finds boundaries only in the image segmented by algorithm. 


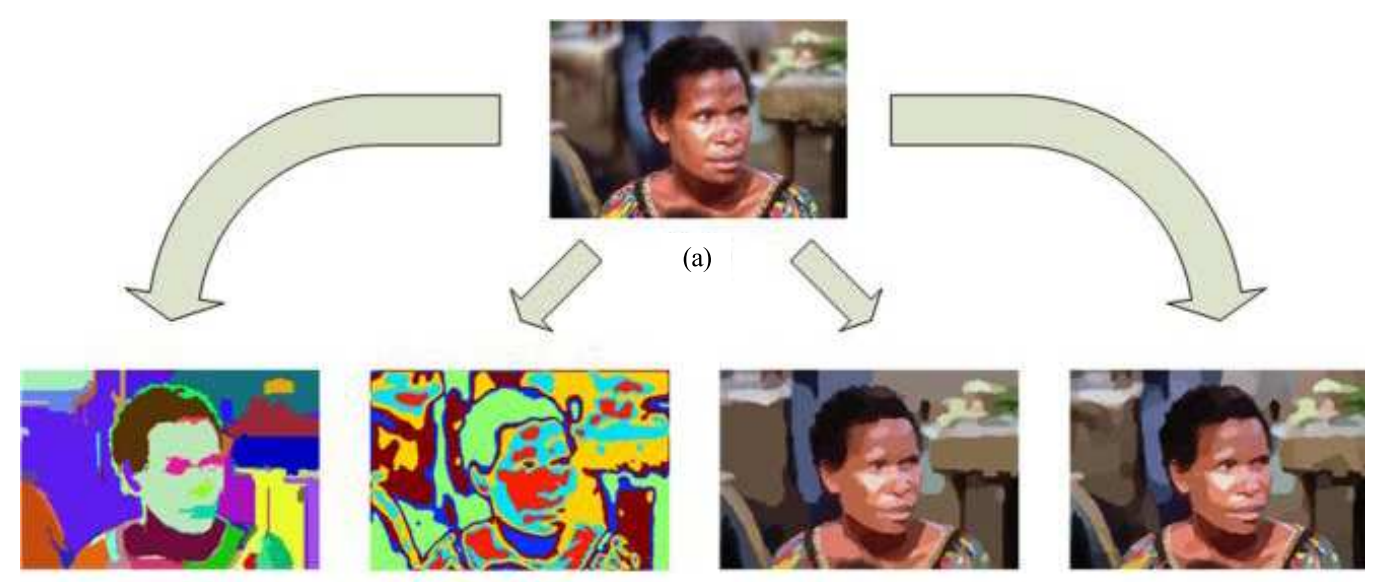

(b)

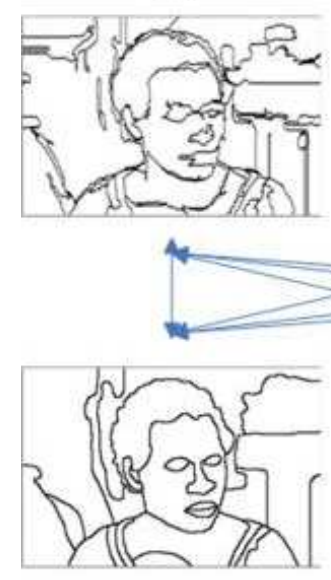

(f) (c)

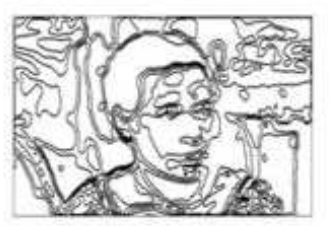

(d)

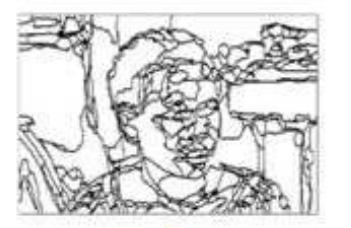

(e)

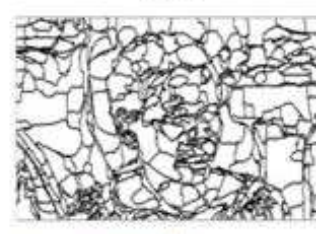

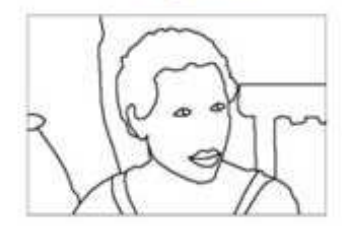

(g)

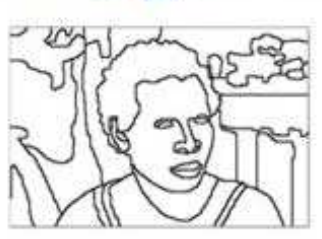

(h)

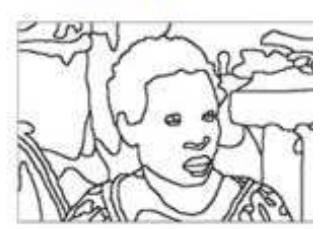

(i)

Fig. 6. Procedure of the using evaluation criterion (a) original image, (b) segments and their boundaries by EGA, (c) segments and their boundaries by KA, (d) segments and their boundaries by MSA (e) segments and their boundaries by BPA (f)-(i) segmentation by human

After that, the boundaries of segments are compared. The algorithm overlays the images and computes the criterion parameters based on the overlay. The computing of values is the last step of algorithm and is given by equations (18-20). The principal block diagram is shown in Fig. 5.

The precision $(P)$, recall $R$ and $F_{1}$ are given by

$$
\begin{aligned}
P & =\frac{C}{C+F} \times 100 \%, \\
R & =\frac{C}{C+M} \times 100 \%, \\
F_{1} & =\frac{2 P R}{P+R} \times 100 \%,
\end{aligned}
$$

where $C$ is the number of correct detected pixels that belongs to boundary, $F$ is the number of false detected pixels and $M$ is the number of not detected pixels. Parameter $F_{1}$ is a combined measure of precision and recall. It is in high values if both, precision and recall have high values and on the other hand, if one of them has low value, the value of $F_{1}$ is going down. Completive procedure of using evaluation criterion is shown in Fig. 6.

\section{EXPERIMENTAL RESULTS}

The aim of image segmentation is to divide image into segments. For segmentation efficiency evaluation, the precision $P$ and recall $R$ of the segments are used [14]. The computing of these parameters and parameter $F_{1}$ were the objective of the experiments.

Experimental part of the paper consists of the experiments on Corel 1000 real image database [15] and experiments on images created by generator. Database A consists of 100 real images from Corel 1000 dataset of images. The size of images from database A is $256 \times 384$ pixels. Examples of these images are shown in Fig. 7.

Database B consists of 100 images created by the image generator. The size of the images from database $\mathrm{B}$ is $500 \times 500$ pixels. Each of the images consists of 6 to 15 objects. The object is inserted into image by different color, size, rotation and angle. Examples of images of database $\mathrm{B}$ are shown in Fig. 8.

In experiments, the optimal values of parameters for each algorithm were used $[6,7,10,13]$. All 200 images were used as input data for segmentation algorithms. The eval- 

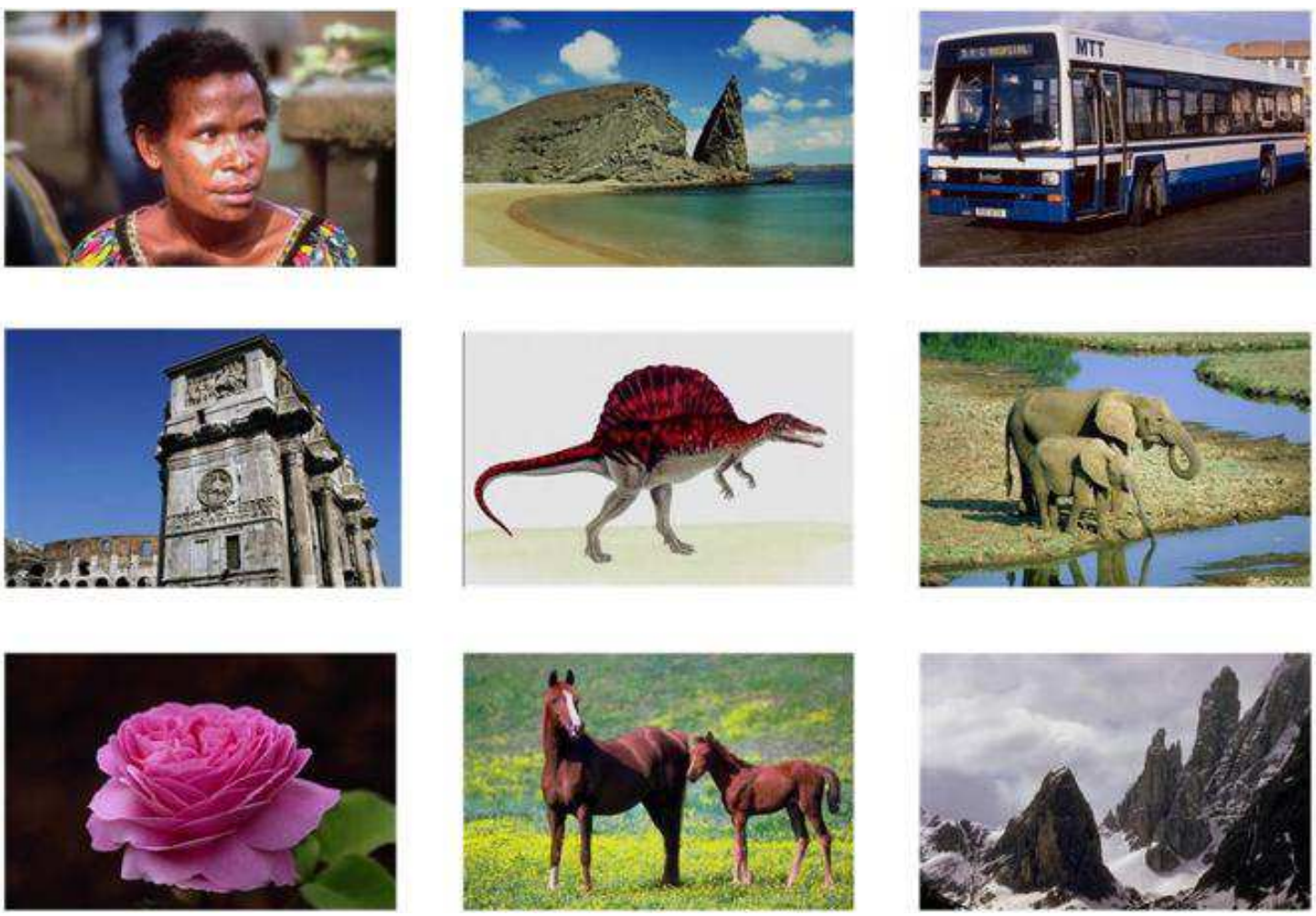

Fig. 7. Example of real images
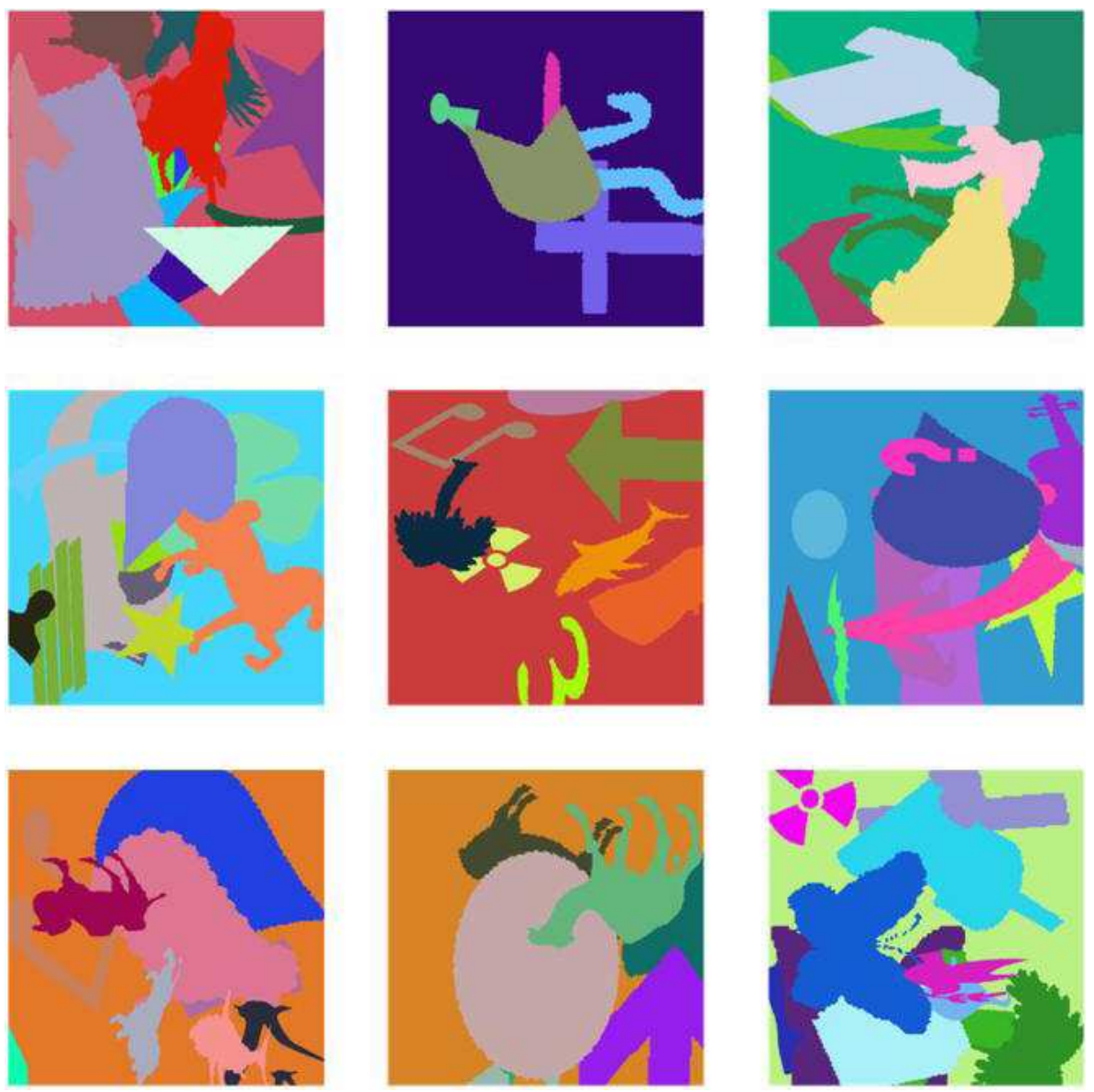

Fig. 8. Example of images created by generator 


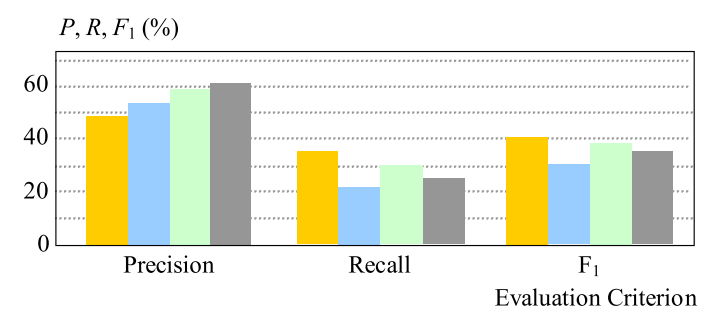

Fig. 9. Avaraged results achieved for real images

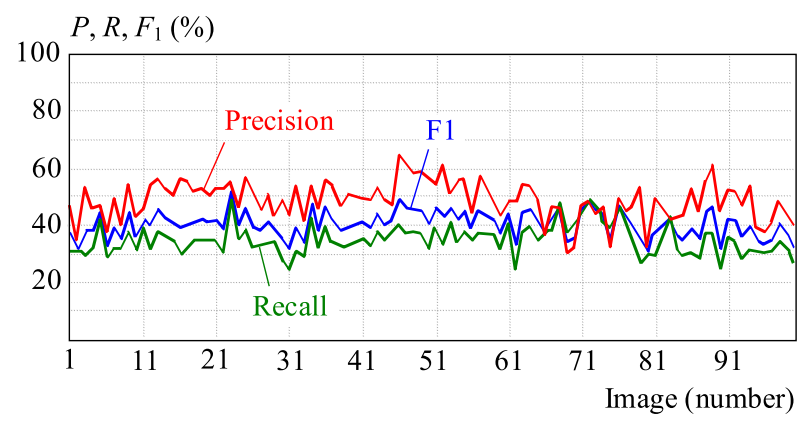

Fig. 10. Results achieved for real images for EGA

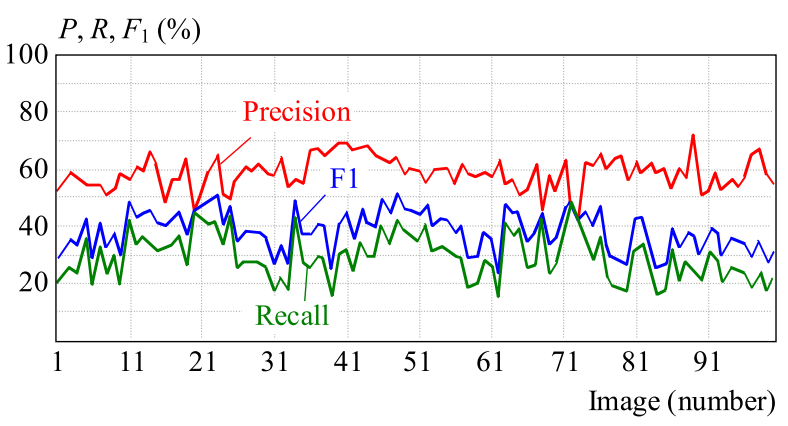

Fig. 12. Results achieved for real images for MSA

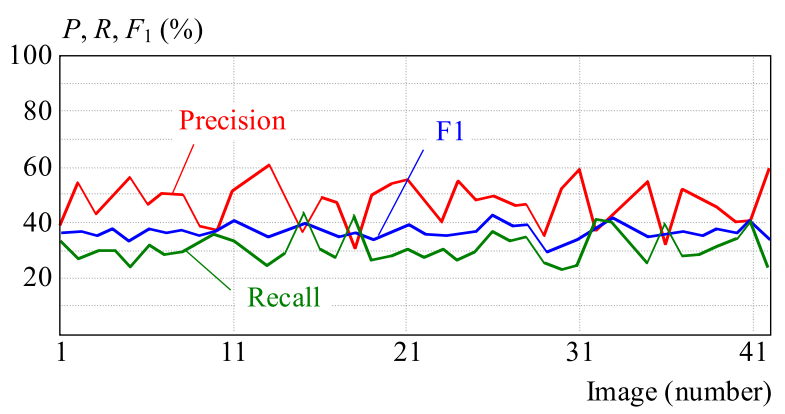

Fig. 14. Results achieved for one image for EGA

uation criterion was applied on the all segmented images. This criterion works by comparison of two images, respectively comparison between boundaries of the segments of image segmented by algorithm and boundaries made by human. The complete procedure for one experiment is shown in Fig. 6.

For real images, the algorithms achieved average results up to $60 \%$ for precision, $35 \%$ for recall and $40 \%$
Table 2. Averaged results achieved for images created by generator

\begin{tabular}{cccccc}
\hline $\begin{array}{c}\text { Type of } \\
\text { algorithm }\end{array}$ & $\begin{array}{c}\text { Number } \\
\text { of images }\end{array}$ & $\begin{array}{c}P \\
(\%)\end{array}$ & $\begin{array}{c}R \\
(\%)\end{array}$ & $\begin{array}{c}F_{1} \\
(\%)\end{array}$ & Score \\
\hline EGA & & 80.63 & 31.39 & 42.16 & 3 \\
KA & \multirow{2}{*}{100} & 61.78 & 33.26 & 42.56 & 4 \\
MSA & & 99.35 & 99.97 & 99.66 & 2 \\
BPA & & 99.46 & 99.94 & 99.70 & 1 \\
\hline
\end{tabular}

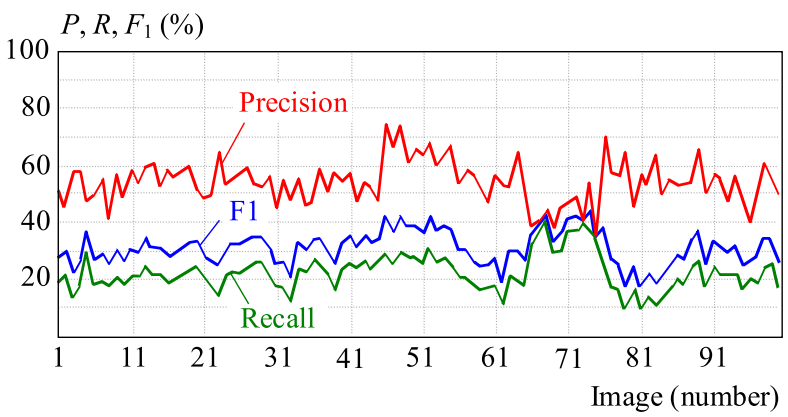

Fig. 11. Results achieved for real images for KMA

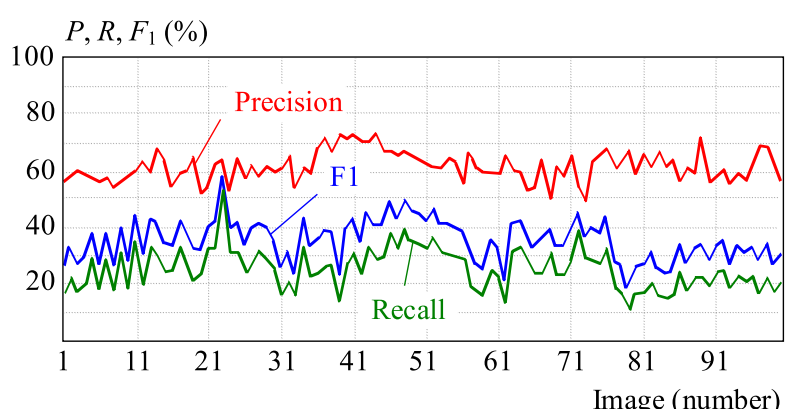

Fig. 13. Results achieved for real images for BPA

for $F_{1}$. No one had best results for all 3 parameters. Algorithm BPA had best results of $\mathrm{P}$ parameter, but insufficient results of $\mathrm{R}$ parameter. Best algorithm of $\mathrm{R}$ parameter is EGA with results about $30 \%$ up to $35 \%$, but lower results of $P$. The final results of $F_{1}$ are up to 40 percent. The average results for all algorithms are shown in Tab. 1 and final results are graphically shown in Fig. 8.

All algorithms achieved better results for images produced by generator. For these images, $k$-means algorithm had average results of $P$ up to $60 \%$, of $R$ up to $33 \%$ and the $F_{1}$ up to $42 \%$. Better results had efficient graph based algorithm with $P$ up to $80 \%, R$ up to $32 \%$ and the $F_{1}$ has about $45 \%$. Best algorithms for images created by generator are MSA and BPA with almost identical results. The algorithms reached values up to $100 \%$. The results are shown in Tab. 2.

The full review of experiments on all real images is shown in Figs. 10-13. The results of EGA method are shown in Fig. 10, KMA method in Fig. 11, MSA method in Fig. 12 and BPA method in Fig. 13. 


\section{CONCLUSION}

In this paper, four well known algorithms and methods for color image segmentation were presented. There were classified and discussed the main definition of all the algorithms. After that, the evaluation criterion of image segmentation was presented. The criterion evaluates and compares segmentation algorithms by a large number of practical experiments. Based on this criterion, the tables and graphs of results were created.

The results from image segmentation algorithms are affected by image factors like color, texture, homogeneity, spatial structure character and many other factors. The best image segmentation algorithm should deal with these factors. The experimental results justify the precision, recall and $F_{1}$ for all algorithms. From the experimental results is obvious that for precision, the best segmentation results had MSA $(P=58.77 \%)$ and BPA $(P=61.41 \%)$. Best algorithm for recall was GBA $(R=35.25 \%)$. Parameter $F_{1}$ has greatest values of $\operatorname{GBA}\left(F_{1}=40.68 \%\right)$.

\section{Acknowledgements}

This paper has been supported by the VEGA grant agency no. 1/0570/10, "Algorithms research for automatic analysis of multimedia data".

\section{REFERENCES}

[1] MORAVČÍK, T.: Image Segmentation in Programming Environment MATLAB, OWD International PhD Workshop, Wisla, 17-20 October 2009, pp. 469-470.

[2] ZHANG, Y. J.: Advances in Image and Video Segmentation, IRM Press, USA, 2006.

[3] ShapiRo, L. G.-STOCKMAN, G. C.: Computer Vision, Prentice Hall, New Jersey, 2001.

[4] SMEulders, A. W. M.-WORRING, M.-SANTINI, S.GUPTA, A.-JAIN, R.: Content-Based Image Retrieval at the End of the Early Years, IEEE Transactions on Pattern Analysis and Machine Intelligence (2000).

[5] BRUZzOnE, L.-CARLiN, L.: A Multilevel Context-Based System for Classification of Very High Spatial Resolution Images, IEEE Transaction on Geoscience and Remote Sensing (2006).

[6] FelenZWAlB, P. F.-HUTTEnlOCheR, D. P.: Efficient Graph-Based Image Segmentation, International Journal of Computer Vision 59 No. 2 (2004).

[7] KANUNGO, T.-MOUNT, D.-NETANYAHU, N.-PIATKO, C.-SILVERMAN, R.-WU, A. Y. : An Efficient $k$-means Clustering Algorithm: Analysis and Implementation, IEEE Transactions and Pattern Analysis and Machine Intelligence 24 No. 7 (2002).

[8] MAT-ISA, N. A.-MASHOR, M. Y.-OTHMAN, N. H. : Comparison of Segmentation Performance of Clustering Algorithms for Pap Smear Images, Proceedings of International Conference on Robotics, Vision, Information and Signal Processing, 2003.

[9] DONG, L.-OGUnBOnA, P.-LI, W.-YU, G.-FAN, L.ZHENG, G. : A Fast Algorithm for Color Image Segmentation, First International Conference on Innovative Computing, Information and Control, vol. ICICIC 06, 2006.

[10] SIQIANG, L.-WEI, L.: Image Segmentation Based on the Mean-Shift in the HSV space, 26th Chinese Control Conference, 2007.
[11] DUDA, R.-HART, P.-STORK, D.: Pattern Classification, second edition, 2000.

12] YEDIDA, J. S.-FREEMAN, W. T.-WEISS, Y.: Understanding Belief Propagation and it is Generalizations, Exploring Artificial Intelligence in the New Millennium, Chap. 8, Jan 2003, pp. 239-236.

13] ZHAOZHENG, Y.-COLLINS, R.: Belief Propagation in a 3D Spatio-Temporal MRF for Moving Object Detection, Computer Vision and Pattern Recognition, CVPR '07. IEEE Conference, 17-22 June 2007, pp. 1-8.

[14] MAKHOUL, J.-KUBALA, F.-SCHQARTZ, R.-WEISCHEDEL, R. : Performance Measures for Information Extraction, In Proceedings of DARPA Broadcast News Workshop, 1999.

[15] Corel 1000 image database. [on line 08.12.2010] Available on the

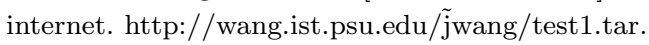

Received 14 February 2011

Peter Lukáč was born in 1984 in Trnava, Slovakia. He received his Ing (MSc) degree in 2008 at the Department of Telecommunications and Multimedia, University of Žilina. Since October 2008 he is PhD. student at the Department of Telecommunications, University of Žilina. His research interest includes digital image processing, research of color image segmentation algorithms.

Róbert Hudec was born in Revúca in 1974, Slovakia. He received his MSc degree in 1998 and his $\mathrm{PhD}$ degree in 2003 both at the Department of Electronics and Multimedia Communications, Technical University in Košice. Nowadays, he is associated professor at the Department of Telecommunications and Multimedia, University of Žilina. His research interests includes digital image processing, filtration of mixed noise by adaptive order-statistics filters, combined low-level image description for MPEG-7 standard.

Miroslav Benčo was born in 1981 in Vranov nad Topl'ou, Slovakia. He received his Ing (MSc) degree in 2005 at the Department of Control and Information Systems and PhD degree in 2009 at department of Telecommunications and Multimedia, University of Žilina. Since January 2009 he is researcher at the Department of Telecommunications, University of Žilina. His research interest includes digital image processing, semantic analysis of multimedia content, image and video classification and retrieval for MPEG-7 standard.

Zuzana Dubcová was born in 1984 in Žilina, Slovakia. She received her Ing. (MSc) degree in 2008 at the Department of Telecommunications and Multimedia, University of Žilina. Since October 2008 she is $\mathrm{PhD}$ student at the Department of Telecommunications, University of Žilina. Her research interest includes digital image processing, examination of algorithm semantic sorting multimedia contents.

Martina Zachariášová was born in Želiezovce, Slovakia, in 1985. She received the Ing (MSc) degree in telecommunications from the University of Žilina, Slovakia, in 2010. Currently, she is PhD. student at Department of Telecommunications and Multimedia at the University of Žilina. Her doctoral work is in the semantic web.

Patrik Kamencay was born in Topol'čany, Slovakia, in 1985. He received the Ing (MSc) degree in telecommunications from the University of Žilina, Slovakia, in 2009. Currently, he is PhD. student at Department of Telecommunications and Multimedia at the University of Žilina. His doctoral work is in the area of 3D image processing. His research interests include holography for 3D display, creating 3D image of the original object. 